\title{
Facharztprüfung - was gilt? (Stand 2007)
}

\author{
Die Kommission für Weiter- und Fortbildung (KWFB) setzt per 1. Januar 2007 \\ die Schwerpunktprüfungen in Gefässchirurgie, Thoraxchirurgie und Phon- \\ iatrie mit eliminatorischer Wirkung in Kraft.
}

\section{Christoph Hänggeli}

Geschäftsleiter Sekretariat Aus-, Weiter- und Fortbildung (AWF)

Korrespondenz: Christoph Hänggeli FMH

Sekretariat Aus-, Weiterund Fortbildung (AWF) Elfenstrasse 18 CH-3000 Bern 15
Per 1. Januar 2007 hat die KWFB in drei weiteren Fachgebieten die eliminatorische Wirkung der Schwerpunktprüfung in Kraft gesetzt. Damit wird jetzt für praktisch alle Facharzttitel und einen Teil der Schwerpunkte das Bestehen der Facharztprüfung bzw. der Schwerpunktprüfung gefordert, selbstverständlich unter Vorbehalt der anwendbaren Übergangsbestimmungen.

\section{Übergangsbestimmungen}

Ausnahmen gelten für Kandidatinnen und Kandidaten, die eine der beiden folgenden Bedingungen erfüllen:

- wer vor dem Inkraftsetzungstermin bereits an einer Facharzt- bzw. Schwerpunktprüfung (ganz oder teilweise) teilgenommen hat, muss keine weitere Prüfung absolvieren;

- wer die Weiterbildung innert zweier Jahre nach dem Inkraftsetzungstermin abgeschlossen hat, benötigt für die Titel- bzw. Schwer- punkterteilung lediglich eine Teilnahmebestätigung über die absolvierte Facharztbzw. Schwerpunktprüfung. Achtung: Sämtliche Voraussetzungen müssen innert zweier Jahre nach dem Inkraftsetzungstermin erfüllt sein (inkl. komplette Facharzt- bzw. Schwerpunktprüfung, ausser wenn die Fachgesellschaft die Zulassung zum 2. Teil wegen ungenügenden 1. Teils verweigert)!

In folgenden Fachgebieten ist weiterhin nur die Teilnahme an der Facharztprüfung gefordert, mit anderen Worten: Das Bestehen der Prüfung ist nicht Voraussetzung für die Erteilung des Facharzttitels.

- Herz- und thorakale Gefässchirurgie;

- Kiefer- und Gesichtschirurgie;

- Medizinische Genetik;

- Nephrologie.

\begin{tabular}{|c|c|c|c|c|c|}
\hline \multicolumn{2}{|c|}{$\begin{array}{l}\text { Tabelle } \\
\text { Muss ich die Facharzt- bzw. Schwerpunktprüfung bestehen? } \\
\text { (Fortsetzung auf der folgenden Seite) } \\
\text { Facharzttitel Inkraftsetzungstermin }\end{array}$} & \multirow[t]{2}{*}{ 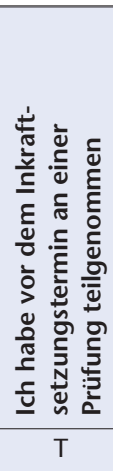 } & 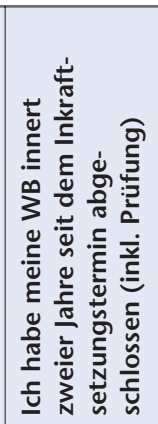 & 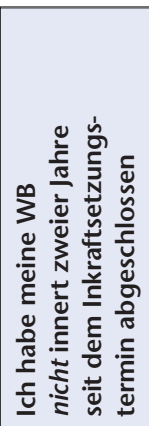 & 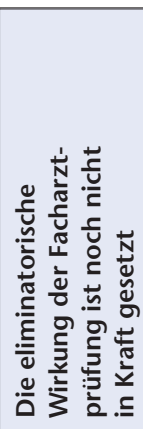 \\
\hline Allergologie und klinische Immunologie & 1.1 .2003 & & $\mathrm{~T}$ & B & - \\
\hline Allgemeinmedizin & 1.1 .2000 & $\mathrm{~T}$ & $\mathrm{~T}$ & B & - \\
\hline Anästhesiologie & 2.4 .1986 & - & - & B & - \\
\hline Angiologie & 1.1 .2005 & $\mathrm{~T}$ & $\mathrm{~T}$ & B & - \\
\hline Arbeitsmedizin & 1.1 .2001 & $\mathrm{~T}$ & $\mathrm{~T}$ & B & - \\
\hline Chirurgie (inkl. Basisexamen) & 1.1 .1999 & $\mathrm{~T}$ & $\mathrm{~T}$ & B & - \\
\hline Dermatologie und Venerologie & 1.1 .2003 & $\mathrm{~T}$ & $\mathrm{~T}$ & B & - \\
\hline Endokrinologie/Diabetologie & 1.1 .2001 & $\mathrm{~T}$ & $\mathrm{~T}$ & B & - \\
\hline Gastroenterologie & 1.1 .2000 & $\mathrm{~T}$ & $\mathrm{~T}$ & B & - \\
\hline Gynäkologie und Geburtshilfe & 1.1 .1999 & $T$ & $\mathrm{~T}$ & B & - \\
\hline Hämatologie & 1.1 .2001 & $\mathrm{~T}$ & $\mathrm{~T}$ & B & - \\
\hline Herz- und thorakale Gefässchirurgie & $1.1 .2008 ?$ & - & - & - & $\mathrm{T}$ \\
\hline nfektiologie & 1.1 .2003 & $\mathrm{~T}$ & $\mathrm{~T}$ & B & - \\
\hline
\end{tabular}


Die gesamte Weiterbildungsdokumentation (mit den aktuellen Prüfungsterminen) ist im Internet unter www.fmh.ch/awf abrufbar. Die Prüfungstermine werden auch in der Schweizerischen Ärztezeitung publiziert. Bei allfälligen Rückfragen steht Ihnen das Sekretariat Aus-, Weiter- und Fortbildung (AWF) der FMH gerne zur Verfügung. Adresse: Postfach 170, Elfenstrasse 18, 3000 Bern 15, Tel. 031 35911 11, Fax 03135911 12, E-Mail: diplome@fmh.ch.

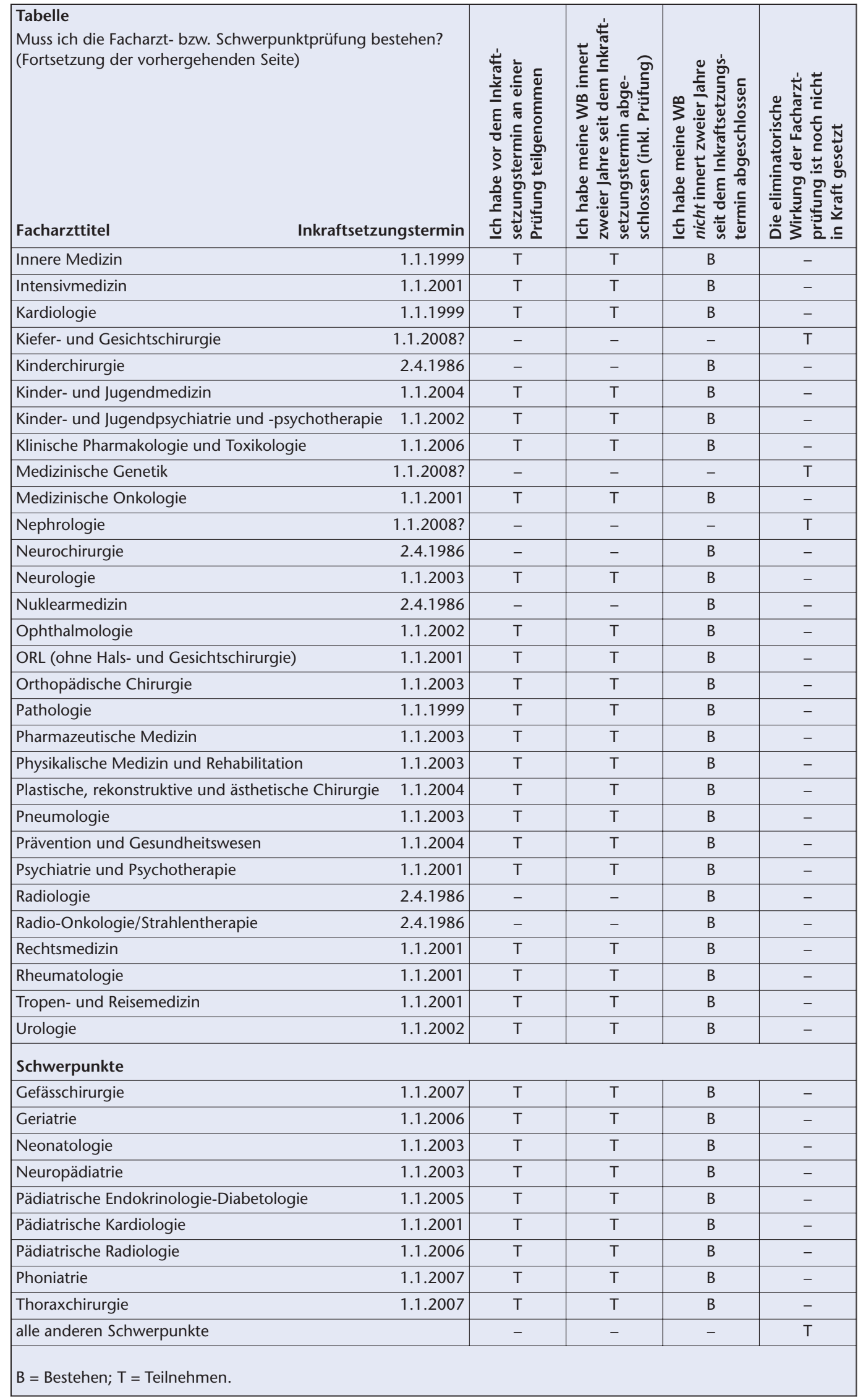

